\title{
Paternité après traitement du cancer du testicule : enquête sur 136 patients
}

\author{
P. Lanfrey, A. Houlgatte, P. Houdelette, P. Berlizot \\ Clinique d'Urologie, Hopital d'Instruction des Armées du Val de Grâce, Paris, France
}

\section{RESUME}

A partir d'un questionnaire adressé à 136 patients suivis de 1981 à 1993 , nous tentons d'évaluer, de façon rétrospective, la paternité aprés traitement du cancer du testicule.

Sur les 76 réponses obtenues, $56 \%$ des patients ont eu au moins un enfant. Aprés traitement, environ 35\% des patients sont considérés comme stériles. Le type de traitement, quel que soit sa modalité, ne semble pas avoir d'influence sur la paternité future. Seulement trois couples, sur les quarante trois patients ayant un désir d'enfant ont eu recours à une procréation médicalement assistée. Au total, 46 grossesses (dont une par fécondation in vitro) ont été obtenues avec un délai moyen de 4 ans par rapport à la fin du traitement.

Nous pensons qu'une étude prospective, en collaboration avec les CECOS apparaît nécessaire pour confirmer ces résultats.

Mots clés : paternité, cancer du testicule

\section{INTRODUCTION}

Le pronostic du cancer du testicule a été considérablement amélioré par l'apparition des nouveaux protocoles de chimiothérapie à base de cis-platine. On rapporte actuellement $95 \%$ de survie à 5 ans pour les patients de stade 1 et 2 et $80 \%$ de survie à 5 ans pour ceux de stade 3 [4]. Les résultats obtenus sur la survie reportent maintenant les préoccupations sur les conséquences physiques et psychologiques de la maladie et de son traitement, et notamment sur la fertilité de cette population jeune, en âge de procréer.

Les données sur la fertilité des patients présentant une tumeur germinale du testicule sont d'interprétation difficile. La plupart des études portent essentiellement sur les caractéristiques du sperme et ne donnent que peu de détails sur la paternité réelle. Il est admis par de nombreux auteurs que 50 à $60 \%$ des patients porteurs d'un cancer testiculaire présentent une altération de la spermatogénèse avant tout traitement complémentaire $[3,6,11,16]$. La qualité du sperme semble toutefois s'améliorer avec le temps et à distance du traitement chez la plupart des patients étudiés [3, 5, 11-13].

La paternité de ces patients, considérés comme subfertiles, reste une donnée difficile a évaluer. Les résultats obtenus sont trés variables d'une étude à l'autre et leur analyse difficile au vu des nombreux paramètres, objectifs et subjectifs, devant être pris en compte.

\section{MATERIELS ET METHODES}

Un enquête retrospective par questionnaire a été réalisée sur 136 patients traités pour cancer du testicule de 1981 à 1993 dans notre 
service. Nous avons exclu préalablement de l'étude les patients âgés de plus de 45 ans, les tumeurs bilatérales et les formes évolutives.

Le questionnaire adressé à chaque patient précisait : l'âge de la partenaire, la situation familiale, l'éventuelle réalisation d'une conservation de sperme au CECOS, le désir de paternité, le nombre d'enfants obtenus avant et aprés traitement du cancer, le délai d'obtention de la première grossesse par rapport à la fin du traitement et au désir de paternité, l'existence d'une éventuelle cause identifiée à la stérilité du couple.

Nous avons obtenu 76 réponses, 30 patients présentaient une tumeur germinale séminomateuse, 46 une tumeur germinale non séminomateuse essentiellement à type de carcinome embryonnaire (19 patients) et de tératocarcinome (12 patients). La répartition de la population en fonction du type histologique et du stade de la tumeur est résumée dans le tableau 1.
Cette cohorte de patients a été divisée en 5 groupes selon le traitement administré :

- groupe 1 : surveillance simple (8 patients).

- groupe 2 : chimiothérapie (13 patients).

dont : $\quad 8: 3$ cycles de BEP.

$4: 4$ cycles d' $E P$.

$1: 1$ cycle de BEP et 2 cycles d'EP.

- groupe 3 : chimiothérapie et curage (28 patients).

dont: 23 : chimiothérapie et curage de masses résiduelles.

5 : curage de stadification et chimiothérapie. (avant 1986).

- groupe 4 : radiothérapie (24 patients).

dont : 19 : radiothérapie dite "prophylactique" (25 GY).

5 : radiothérapie dite "curatrice" (35 à 40 GY).

- groupe 5 : curage isolé de stadification (3 patients). (avant 1986).

Tableau 1 : Répartition de la population en fonction du type histologique et du stade.

\begin{tabular}{|c|c|c|c|c|c|c|c|}
\hline & \multirow{2}{*}{$\begin{array}{c}\text { Nombre } \\
\text { de patients }\end{array}$} & \multirow[t]{2}{*}{ Stade I } & \multicolumn{3}{|c|}{ Stade II } & \multirow[t]{2}{*}{ Stade III } & \multirow[t]{2}{*}{ Stade IV } \\
\hline & & & $\mathbf{A}$ & B & $\mathbf{C}$ & & \\
\hline Séminome & 30 patients & 19 & 6 & $\begin{array}{c}1 \\
\text { Tot. } 10\end{array}$ & 3 & 1 & 0 \\
\hline $\begin{array}{l}\text { Carcinome } \\
\text { embryonnaire }\end{array}$ & 19 patients & 5 & 5 & $\begin{array}{c}5 \\
\text { Tot. } 10\end{array}$ & 0 & 3 & 1 \\
\hline Térato-carcinome & 12 patients & 6 & 2 & $\begin{array}{c}1 \\
\text { Tot. } 4\end{array}$ & 1 & 0 & 2 \\
\hline Chorio-carcinome & 3 patients & 3 & & 0 & & 0 & 0 \\
\hline \multicolumn{8}{|l|}{ Chorio-carcinome } \\
\hline + carc. embry. & 4 patients & 1 & 1 & $\begin{array}{c}1 \\
\text { Tot. } 2\end{array}$ & 0 & 0 & 1 \\
\hline + tératome & 3 patients & 1 & 0 & $\begin{array}{c}1 \\
\text { Tot. } 1\end{array}$ & 0 & 0 & 1 \\
\hline + térato-carc. & 4 patients & & 0 & $\begin{array}{c}2 \\
\text { Tot. } 3\end{array}$ & 1 & 0 & 0 \\
\hline Teratome mature & 1 patient & 0 & 1 & $\begin{array}{c}0 \\
\text { Tot. } 1\end{array}$ & 0 & 0 & 0 \\
\hline
\end{tabular}




\section{RESULTATS}

Sur les 76 réponses parmi les 136 questionnaires envoyés, 20 patients avaient déjà eu au moins un enfant avant l'orchidectomie. Un dépot de sperme au CECOS avait été effectué par 42 d'entre-eux.

43 patients d'âge moyen 32 ans (21-42 ans) avaient un désir de paternité. 28 d'entreeux ont eu au moins un enfant avec un délai moyen pour l'obtention de la première grossesse de 3,7 ans (1 à 6 ans) par rapport à la fin du traitement. Le délai par rapport au désir de paternité varie de 1 mois à 5 ans (moyenne 1,7 ans).

Sur les 46 grossesses obtenues, 39 ont été menées à terme, 7 fausses couches sont à déplorer. Le recours à la procréation médicalement assistée concerne trois couples.

Bien que le nombre de patients dans chaque catégorie soit faible il ne semble pas exister de différence significative dans l'obtention d'une grossesse entre les différentes options thérapeutiques concernant la tumeur testiculaire (Tableau 2).

Parmi les 15 couples n'ayant pas eu d'enfants l'origine de la stérilité était considérée comme "masculine" dans 8 cas, féminine dans 1 cas (obstruction tubaire bilatéra- le), non précisée dans 6 cas. Un patient ayant fait l'objet d'un curage de masse résiduelle présentait une éjaculation rétrograde.

33 patients, d'âge moyen 35 ans, ne souhaitent pas d'enfant après traitement de la tumeur. Il s'agit essentiellement de patients ayant fait l'objet d'une radiothérapie ou d'un traitement lourd associant chimiothérapie et éxérèse des masses résiduelles rétropéritonéales (Tableau 3 ). La raison évoquée à cette absence de désir de paternité étant pour 13 d'entre-eux, d'âge moyen 40 ans (23-45 ans), une procréation antérieure. Pour les 20 autres, d'âge moyen 25 ans (18-32 ans), le statut marital est retenu (15 célibataires).

\section{DISCUSSION}

La plupart des études réalisées s'attachent à l'analyse des effets de la maladie et de son traitement sur la spermatogénèse. Il est reconnu que la majorité des traitements utilisés dans le cancer du testicule altèrent la qualité du sperme [10]. Celle-ci semble toutefois s'améliorer avec le temps et à distance du traitement chez 40 à $75 \%$ des patients $[10,11]$. Mais cette amélioration "biologique", ne permet pas de juger, à elle seule, de la fertilité réelle.

Tableau 2 : Population désirant un enfant.

\begin{tabular}{lccccc}
\hline & Nombre patients & $\begin{array}{c}\text { Enfants } \\
\text { Avant } \\
\text { traitement }\end{array}$ & $\begin{array}{c}\text { Enfants } \\
\text { Après } \\
\text { traitement }\end{array}$ & $\begin{array}{c}\text { Enfants } \\
\text { Avant + Après } \\
\text { traitement }\end{array}$ & Pas d'enfants \\
\hline Surveillance & 7 patients & 0 & 4 & 1 & 2 \\
Chimiothérapie & 9 patients & 0 & 4 & 2 & 3 \\
$\begin{array}{l}\text { Chimiothérapie } \\
\text { Curage }\end{array}$ & 13 patients & 0 & 7 & 1 & 5 \\
Radiothérapie & 12 patients & 0 & $(1$ FIV) & & 4 \\
Curage & 2 patients & 0 & 7 & 1 & 1 \\
\hline
\end{tabular}




\begin{tabular}{lccc}
\hline & Nombre de patients & Enfants avant traitement & Pas d'enfant \\
Surveillance & 1 patient & 0 & 1 \\
Chimiothérapie & 4 patients & 1 & 3 \\
Chimiothérapie curage & 15 patients & 6 & 9 \\
Radiothérapie & 12 patients & 8 & 4 \\
Curage isolé & 1 patient & 0 & 1 \\
\hline
\end{tabular}

Les données disponibles sur la paternité des patients ayant présenté un cancer du testicule sont rares et trés variables.

Avant traitement, la population de sujets oligospermiques varie selon les auteurs de 25 à $75 \%[3,6,10,16]$. La plupart d'entreeux évaluent la fertilité sur les données du spermogramme. De ce fait, Lange [10] considère que $60 \%$ des sujets sont subfertiles au moment du diagnostic. Hansen [8] et Johnson [9] évaluent, quant à eux, à environ $10 \%$ le nombre de sujets que l'on peut considérer comme infertiles.

Aprés traitement, selon les auteurs 30 à $53 \%$ des sujets souhaitant un enfant sont infertiles quel que soit le type de traitement $[1,2,8]$. La paternité de ces patients est également trés variable selon les séries. Pour Aass [1] et Hansen [8], 70\% d'entreeux environ ont eu au moins 1 enfant, $30 \%$ environ pour Gritz [7], sans que cette différence puisse s'expliquer par le stade évolutif ou le type de traitement subi.

Les résultats obtenus en fonction des traitements semblent eux aussi discordants. Il ne semble pas y avoir pour Hansen [8] de différence significative dans les différentes sous-populations en fonction du traitement utilisé (65\% de sujets fertiles). Les résultats publiés par Fossa [1] sur 149 patients semblent différents. En dehors du curage rétropéritonéal bilatéral étendu responsable d'une éjaculation rétrograde chez 80 à $90 \%$ des sujets, les traitements associant radiothérapie et chimiothérapie sont responsables d'une infertilité chez $78 \%$ des patients. Selon Fossa, l'altération de la fertilité varie avec l'intensité et le type de traitement utilisé. On retrouve, dans cette étude, environ $20 \%$ de sujets infertiles dans les groupes de patients ayant subit un curage limité ou une radiothérapie isolée ( $\max$ $40 \mathrm{GY}$ ) et environ $65 \%$ de sujets infertiles aprés chimiothérapie isolée ou associée à un curage de masses résiduelles. Cette série prend en compte des thérapeutiques sans relation avec les protocoles en vigueur actuellement, tant en ce qui concerne l'étendu du curage que la chimiothérapie et l'intensité de la radiothérapie.

L'interprétation de l'ensemble de ces données apparaît complexe, de nombreux paramètres influant sur la reproduction, devant être pris en compte indépendament des différents résultats publiés (désir de paternité, âge moyen de la population, statut marital, délai considéré par rapport au désir de paternité, origine de l'infertilité ...). A ces paramètres "objectifs", s'ajoutent les effets de la maladie et de son traitement sur la fonction sexuelle des patients. Schrover [14, 15] estime que 10 à $40 \%$ des patients traités pour cancer du testicule présentent des séquelles "psycho-sexuelles" à type d'éjaculation prématurée, de diminution de la libido, d'anorgasmie, ou de troubles de l'érection. 
Il apparaît donc difficile d'appréhender dans son intégralité la fertilité d'un couple en tenant compte de l'ensemble des facteurs pouvant avoir une incidence sur celleci. L'enquête rétrospective par questionnaire tentant d'évaluer la paternité de 136 patients suivis aprés traitement pour cancer du testicule cherche à tenir compte de ces facteurs.

Sur les 76 patients ayant répondu à notre enquête, $56 \%$ d'entre-eux ont eu au moins un enfant. Ce résultat se rapproche de ceux obtenus au cours de deux enquêtes rétrospectives par Hansen (65\%) [8] et Fossa (70\%) [1] sur respectivement 177 et 149 patients. Après traitement, dans la population étudiée, environ $65 \%$ des patients ayant un désir de paternité ont eu un enfant. Bien que le nombre de patients désirant un enfant par sous-groupe de traitement soit faible, il ne semble pas y avoir de différence significative de paternité selon le traitement administré. A noter deux points dans la population des patients désirant un enfant : tous les patients ayant eu un enfant avant le traitement en ont eu un après quel que soit le traitement administré (Tableau 2), aucun des patients ayant subit une radiothérapie dite "curatrice" (supérieure à 35 GY) n'a eu d'enfant. Au total, 15 patients désirant un enfant n'en ont pas eu à distance du traitement. La cause de l'infertilité, quand elle est connue semble être majoritairement masculine. Le délai moyen d'obtention de la première grossesse par rapport au désir de paternité ne semble pas différent du délai de 2 ans considéré comme normal dans une population standard. Le recours à la procréation médicalement assistée est faible, seulement trois couples ont tenté une fécondation in vitro malgré l'absence de grossesse aprés un délai moyen de désir de paternité d'environ 4,5 ans ( 2 ans- 8 ans).

L'analyse de la population des 33 patients ne désirant pas d'enfant retrou- ve deux sous-groupes distincts. Une sous-population de patients dont l'âge moyen est de 40 ans ayant déjà eu au moins un enfant avant le traitement (13 patients), et une sous-population de patients plus jeunes, majoritairement célibataires dont l'âge moyen est de 25 ans (20 patients).

\section{CONCLUSION}

L'analyse de la littérature rend compte de la difficulté que l'on peut avoir à recueillir de façon rétrospective des données sur la fécondité. La variabilité des résultats obtenus selon les études, et les nombreux paramètres qui doivent être pris en compte, rendent les synthèses difficiles.

L'interprétation des résultats de cette étude doit être prudente en raison d'une part, du biais de sélection qu'engendre ce type d'enquête rétrospective par questionnaire et d'autre part, en raison du nombre important de sujets perdus de vue (environ $40 \%$ ). Elle ne saurait être qu'une donnée supplémentaire sur la fertilité des patients aprés traitement du cancer du testicule et ne constitue qu'une base en vue d'une étude prospective future effectuée en collaboration avec les CECOS assurant le suivi de leurs conservation. Celle-ci permettrait d'appréhender de façon plus précise et plus exacte la fertilité réelle de cette population afin de pouvoir informer de façon plus exacte ces patients jeunes et souvent anxieux sur leur fertilité future et, peut-être, leur sexualité.

Il faut néammoins souligner les points suivants :

- le délai moyen de 2 ans pour obtenir une première grossesse par rapport au désir de paternité.

- l'absence de recours à une procréation médicalement assistée chez 15 couples n'ayant pas obtenu une paternité après un délai moyen d'environ 4 ans. 


\section{REFERENCES}

1. AASS N., KAASA S., LUND E., KAALHUS O., SKARD-HEIER M., FOSSA S.D. : Long-term somatic side-effects and morbidity in testicular cancer patients.Br. J. Cancer 1990, 61 : 151155.

2. BERTHELSEN J.G. : Testicular cancer and fertility. Int. J. Androl. : 1987, $10: 371-380$.

3. CARROL P.R., MORSE M.J., WITHMORE W.F. : Fertility status of patients with clinical stage 1 testes tumors on a surveillance protocol. J. Urol $1987,138: 70-72$.

4. CARTER M. : Fertility in the testicular cancer patient. World J Urol 1993, $11: 70-75$.

5. DRASGA R.E., EINHORN L.H., WILLI Eur Urol 1984, $10: 173-177$.

6. FOSSA S.D., ABYHOLM T., AAKVAAG A. : Spermatogenesis and hormonal status after orchiectomy for cancer and before supplementary treatment. Eur. Urol. 1984, 10 : 173-177.

7. GRITZ E.R., WELLISH D.K., WANG H., SIAU J., LANDSVERK J.A., COSGROVE MD. : Longterm effects of testicular cancer on sexual functionning in married couples. Cancer, 1987, 64 : 1560-1567

8. HANSEN P.V., GLAVIND K., PANDURO J., PEDERSEN M. : Paternity in patients with testicular germ cell cancer : Pretreatment and posttreatment findings. Eur. J. Cancer. 1991, Vol 27, $11: 1385-1389$.

9. JOHNSON D.E., FUEGER J.J., ALFARO P.J., SPITZ M.R., NEWELL G.R. : Subfertility : an etiologic factor in development of testicular cancer. Urol, 1987, Vol 30, $3: 199-201$.

10. LANGE P.H., CHANG W.Y., FRALEY E.E. : Fertility issues in the therapy of nonseminomatous testicular tumors. Urol Clin North Am 1987, 14 : 731746 .

11. LANGE P.H., NARAYAN P., VOGELZANG N.J, SCHAFER R.B., KENNEDY B.J., FRALEY R.B. : Return of fertility after treatment for nonseminomatous testicular cancer: Changing concept. J. Urol. 1982, $129: 1131-1135$.

12. NIJMAN J.M., SCHAFFORDT-KOOPS H., KREMER J. : Gonadal function after surgery and chemotherapy in men with stage 2 and 3 nonseminomatous testicular tumor. J. Clin. Oncol. 1987, 5 : 651-656.

13.PRESTI J.C., HERR H.W., CARROLL P.R. : Fertility and testis cancer. Urol. Clin. North. Am. 1993, Vol 20, 1: 173-179.
14. SCHOVER L.R., GONZALES M., Von ESCHENBACH A.C. : Sexual and marital relationships after radiotherapy for seminoma. Urol 1986, Vol $27,2: 117-123$.

15. SCHOVER L.R., Von ESCHENBACH A.C. : Sexual and marital relationships after treatment fot nonseminomatous testicular cancer. Urol 1985, Vol 25, $3: 251-255$

16. THACHIL J.W., JEWETT M.A.S. RIDER W.D. : The effects of cancer and cancer therapy on male fertility.J. Urol. 1981, $126:$ 141-145.

\section{ABSTRACT}

Paternity after treatment of testicular cancer
P. Lanfrey, A. Houlgatte,
P. Houdelette, P. Berlizot

We sent a questionnaire to 136 patients, treated between 1981 and 1993 , to try to evaluate, retrospectively, fertility amongst patients following treatment for testicular cancer.

From the 76 complete questionnaires, $56 \%$ of patients had fathered at least one child. After treatment, approximately $35 \%$ of patients were steriles. Treatment, in wich ever form, does not seem to influence fertility. Only 3 couples resorted to infertility treatment. There were 46 pregnancies in total, one of wich resulted from IVF, on average 4 years after the end of treatment for testicular cancer.

A prospective study carried out in conjonction with the centre for study and conservation of sperm would seem to be neceessary to consolidate these findings.

Keys Words : Paternity, Testicular cancer 